\title{
Análise do uso de materiais de biossegurança por profissionais da área da saúde no ambiente hospitalar: uma revisão integrativa
}

\author{
Analysis of the use of biosafety materials by health professionals in the hospital \\ environment: an integrative review
}

Análisis del uso de materiales de bioseguridad por parte de profesionales de la salud en el entorno hospitalario: una revisión integradora

\begin{abstract}
Maria Williane Batista Magalhões ${ }^{1 *}$, Rayane dos Santos Lopes ${ }^{1}$, Ângela Letícia Silva Lima ${ }^{1}$, Francisca Taysa de Abreu Silva ${ }^{1}$, Hellen Dayanne Pereira Silva ${ }^{1}$, Milka Kellen Maciel Leite ${ }^{1}$, Jessica Caroline Sousa Carvalho ${ }^{1}$, Nádyla Paloma Lima Vila ${ }^{1}$, Anderson Maciel dos Anjos Lopes ${ }^{2}$, Eduardo Kellyton de Oliveira Costa ${ }^{1}$.
\end{abstract}

\section{RESUMO}

Objetivo: Verificar na literatura o conhecimento de profissionais da área da saúde acerca do uso de matérias de biossegurança no ambiente hospitalar. Métodos: Trata-se de revisão integrativa, onde foram investigados estudos publicados nas bases de dados eletrônicas no período de janeiro de 2014 à setembro de 2019, com os descritores "Biossegurança"; "Hospital"; "Profissional de saúde"; "Prevenção de Acidentes" e "Serviço Hospitalar de Admissão de Pacientes. Após a seleção de acordo com os critérios, cinco estudos foram utilizados. Resultados: A literatura aponta, que além da falta de conhecimento e materiais adequados, o manuseio de forma incorreta dos uso dos Equipamentos de Proteção Individuais dificultam a adesão dos profissionais da saúde no âmbito hospitalar. Considerações Finais: Estudos mostram que os profissionais da saúde precisam de conhecimentos sobre os riscos que são expostos no ambiente de trabalho, bem como conscientização para o uso adequado minimizando acidentes ocupacionais e reduzindo o número de infecções.

Palavras-chave: Biossegurança, Hospital, Profissional de saúde, Prevenção de acidentes, Serviço hospitalar de admissão de pacientes.

\begin{abstract}
Objective: To verify in the literature the knowledge of health professionals about the use of biosafety materials in the hospital environment. Methods: This is an integrative review, which investigated studies published in electronic databases from January 2014 to September 2019, with the descriptors "Biosafety"; "Hospital"; "Healthcare professional"; "Accident Prevention" and "Hospital Patient Admission Service. After selection according to the criteria, five studies were used. Results: The literature points out that, in addition to the lack of knowledge and adequate materials, incorrect handling of the use of Personal Protective Equipment makes it difficult for health professionals to adhere to the hospital environment. Final Considerations: Studies show that health professionals need knowledge about the risks they are exposed to in the work environment, as well as awareness for proper use, minimizing occupational accidents and reducing the number of infections.
\end{abstract}

Key words: Biosafety, Hospital, Health professional, Accident prevention, Hospital service for patient admission.

${ }^{1}$ Centro Universitário Santo Agostinho (UNIFSA), Teresina - PI. *E-mail: mariawillianebatista@gmail.com

${ }^{2}$ Associação de Ensino Superior do Piauí (AESPI), Teresina - PI. 


\section{RESUMEN}

Objetivo: Verificar en la literatura el conocimiento de los profesionales de la salud sobre el uso de materiales de bioseguridad en el ámbito hospitalario. Métodos: Se trata de una revisión integradora, que investigó estudios publicados en bases de datos electrónicas desde enero de 2014 a septiembre de 2019, con los descriptores "Bioseguridad"; "Hospital"; "Profesional de la salud"; "Prevención de Accidentes" y "Servicio de Admisión de Pacientes Hospitalarios. Después de la selección según los criterios, se utilizaron cinco estudios. Resultados: La literatura señala que, además del desconocimiento y de los materiales adecuados, el manejo incorrecto del uso de los Equipos de Protección Individual dificulta que los profesionales de la salud se adhieran al ambiente hospitalario. Consideraciones finales: Los estudios demuestran que los profesionales de la salud necesitan conocimiento sobre los riesgos a los que están expuestos en el entorno laboral, así como conciencia del uso adecuado, minimizando los accidentes laborales y reduciendo el número de infecciones.

Palabras clave: Bioseguridad, Hospital, Profesional de la salud, Prevención de accidentes, Servicio hospitalario de admisión de pacientes.

\section{INTRODUÇÃO}

Biossegurança diz respeito a um conjunto de ações destinadas a prevenir, minimizar ou eliminar riscos que podem comprometer a saúde do homem, dos animais, do meio ambiente ou qualidade dos trabalhos desenvolvidos (PAPINI TS, et al., 2014). No ambiente hospitalar é necessário seja adotado à risca a implementação das medidas de biossegurança, tanto os profissionais da saúde quanto os pacientes estão expostos à risco de contágio por agentes infecciosos (ARAÚJO DL e OLIVEIRA JL, 2020).

Foram adotadas as precauções universais para esses trabalhadores, como a Norma Regulamentadora do Ministério do Trabalho (NR32), que dispõe da segurança no trabalho em serviços da saúde (MOTA EC, et al., 2014). Estas medidas de biossegurança fornecem melhorias na diminuição de custos, na qualidade do atendimento e nas doenças adquiridas da prática no ambiente hospitalar, tanto para os profissionais ali presente como para os clientes e seus familiares (SILVA PIRES YM, et al., 2019).

Profissionais da área da saúde estão constantemente suscetíveis a acidentes com material biológico e são os principais envolvidos em acidentes de trabalho, isso se dá, pela complexidade das atividades executadas na prestação dos cuidados e condições laborais e baixa adesão dos indivíduos às estratégias de biossegurança (ARANTES MC, et al., 2017).

Isso pode estar relacionado, majoritariamente, ao fato de muitos profissionais não considerarem necessário os equipamentos de proteção individual sejam por desconforto decorrente do uso, por considerar que o mesmo atrapalha a execução de procedimentos, por não haver material de proteção suficiente, ou ainda, por não saber o real risco ao qual estão expostos (CASTRO MR, et al., 2013).

Na assistência à saúde, o risco biológico considerado o maior risco, é responsável pelos agravos sofridos por profissionais da saúde e se relaciona às peculiaridades das tarefas executadas nas suas atividades laborais (REZENDE KCAD, et al., 2012).

As medidas de Precaução padrão aplicam-se a qualquer paciente, independentemente, do diagnóstico clínico ou sorológico e Equipamentos de Proteção Individual (EPI), como luvas de procedimento, aventais, máscaras cirúrgicas e protetores oculares são recomendados sempre que o contato com fluidos corporais for previsto. Reforçam, tais medidas, a recomendação de higienização das mãos, antes e após o contato com pacientes e fluidos orgânicos, o descarte de materiais perfuro cortantes em recipientes rígidos, cuidados com reprocessamento de materiais e administração de medicamentos injetáveis (MALAGUTI-TOFFANO SE, et al., 2015).

Em vista aos constantes acidentes de trabalho, foi criada a Norma Regulamentadora 32 (NR 32), que aborda a segurança e saúde no trabalho em serviços de saúde através da Portaria $n^{\circ} 485$, de 11 de novembro 
de 2005. O objetivo dela é estabelecer diretrizes básicas para que as organizações e estabelecimentos de saúde possam adotar medidas de proteção à segurança e à saúde dos profissionais de saúde, assim como daqueles que desempenham atividades de promoção e assistência à saúde em geral (CUNHA AC e MAURO MYC, 2010).

O uso adequado, correto e frequente de EPIs pelos profissionais, associada à higienização das mãos e ao seguimento de técnicas assépticas nos procedimentos invasivos, desempenham um importante papel na redução do risco de infecção, bem como, para sua própria proteção (SOUZA ELV, et al., 2011; GARCEZ GO, et al, 2019; CASTRO MR, et al., 2013).

Os fatos acima indicados despertaram um importante interesse exponencial em investigar o conhecimento e a atenção sobre biossegurança entre os profissionais da saúde, pois tem-se observado diferentes procedimentos com ausência de EPI'S, vestuário inadequado, reencape de agulhas, constante uso de caixas de perfurocortantes, com material acima do limite permitido. Deste modo, o presente estudo teve como objetivo verificar na literatura o conhecimento de profissionais da área da saúde acerca do uso de matérias de biossegurança no ambiente hospitalar.

\section{MÉTODOS}

Esta revisão integrativa investigou estudos publicados em bases de dados eletrônicas no período entre janeiro de 2014 e junho de 2019, utilizando trabalhos que abordassem o conhecimento de profissionais sobre a importância do conhecimento dos profissionais da saúde sobre uso dos materiais de biossegurança no ambiente hospitalar. Para isso foram realizadas consultas e pesquisas nas bases de dados científicos: Medical Literature Analysis and Retrieval System Online (Pubmed/Medline), Scientific Electronic Library Online (Scielo) e Literatura Latino-americana e do Caribe em Ciências da Saúde (Lilacs).

$\mathrm{Na}$ busca do estudo foram pesquisados os seguintes termos descritores "Biossegurança"; "hospital"; "Profissional de saúde"; "Prevenção de Acidentes" e "Serviço Hospitalar de Admissão de Pacientes". A pesquisa foi realizada no período de outubro a dezembro de 2019, sendo selecionados artigos de ensaio clínico, estudo de coorte e transversal, sendo utilizados os idiomas português e inglês, foram selecionados os publicados nos 5 últimos anos (de 2014 a 2019). Todos os artigos foram analisados por 9 pesquisadores, inicialmente foram feitas as análises dos título e, posteriormente, a leitura crítica do resumo para aplicação dos critérios de inclusão. Dentro dos critérios de inclusão, estabeleceram-se artigos originais completos, no qual tivessem relação com o presente estudo, seja que se adequassem a temática "Uso de materiais de biossegurança por profissionais da área da saúde no ambiente hospitalar".

Após a seleção dos artigos, conforme os critérios de inclusão, foi realizada a leitura de todos eles, dos quais se retiraram as informações de interesse, como também, foram investigadas suas listas de referências, visando encontrar artigos adicionais para a construção da revisão.

Entraram como critérios de exclusão os artigos de revisão de literatura, teses e dissertações, assim como àquele cujo texto completo não fosse disponível até após busca em biblioteca de referência, ou seja, aqueles cuja aquisição, por algum problema técnico, não fosse possível. Com os artigos selecionados, foram tabuladas as principais variáveis de interesse, como o tamanho da amostra, o objetivo, a metodologia, os parâmetros avaliados e os principais resultados.

\section{RESULTADOS}

Inicialmente, foram encontrados 88 artigos (Pubmed $=32$; Scielo $=19$; Lilacs $=37$ ). Desses, 22 estavam duplicados nas referidas bases de dados, restando 66 artigos para a leitura dos títulos, dos quais 37 foram excluídos por não serem adequados aos critérios determinados. 29 artigos selecionados para análise dos resumos, 21 foram excluídos, restando apenas 8 para participar deste estudo. Após revisão das referências destes, nenhum outro trabalho foi incorporado. Ao fim das buscas de dados, a revisão foi composta por oito artigos, e o fluxograma da Figura 1 detalha todos os procedimentos de busca pelas pesquisas selecionadas para a confecção desta revisão. 
Figura 1 - Fluxograma dos estudos selecionados.

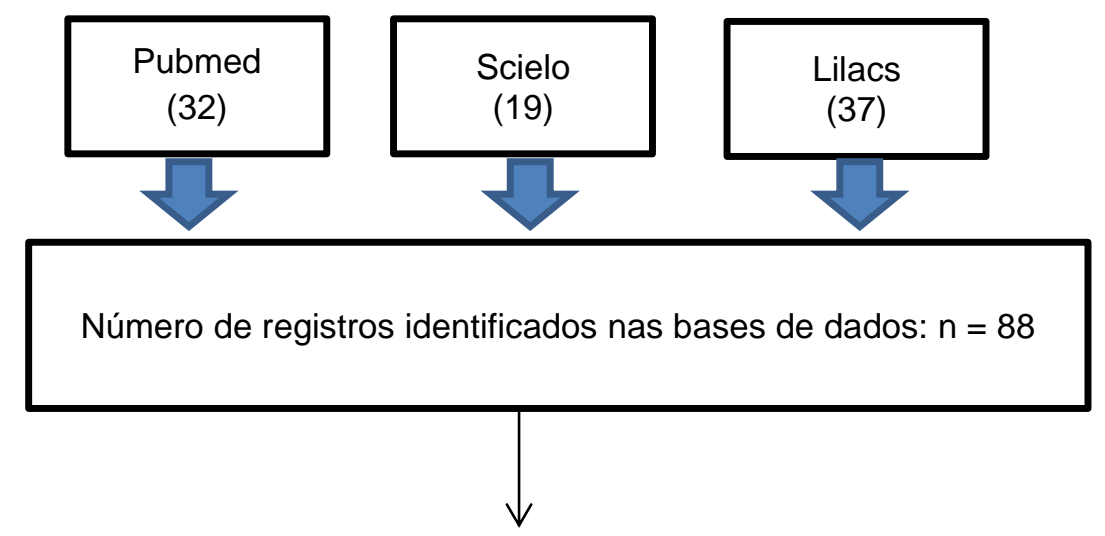

Número de artigos excluídos após apresentarem duplicidade: $\mathrm{n}=22$

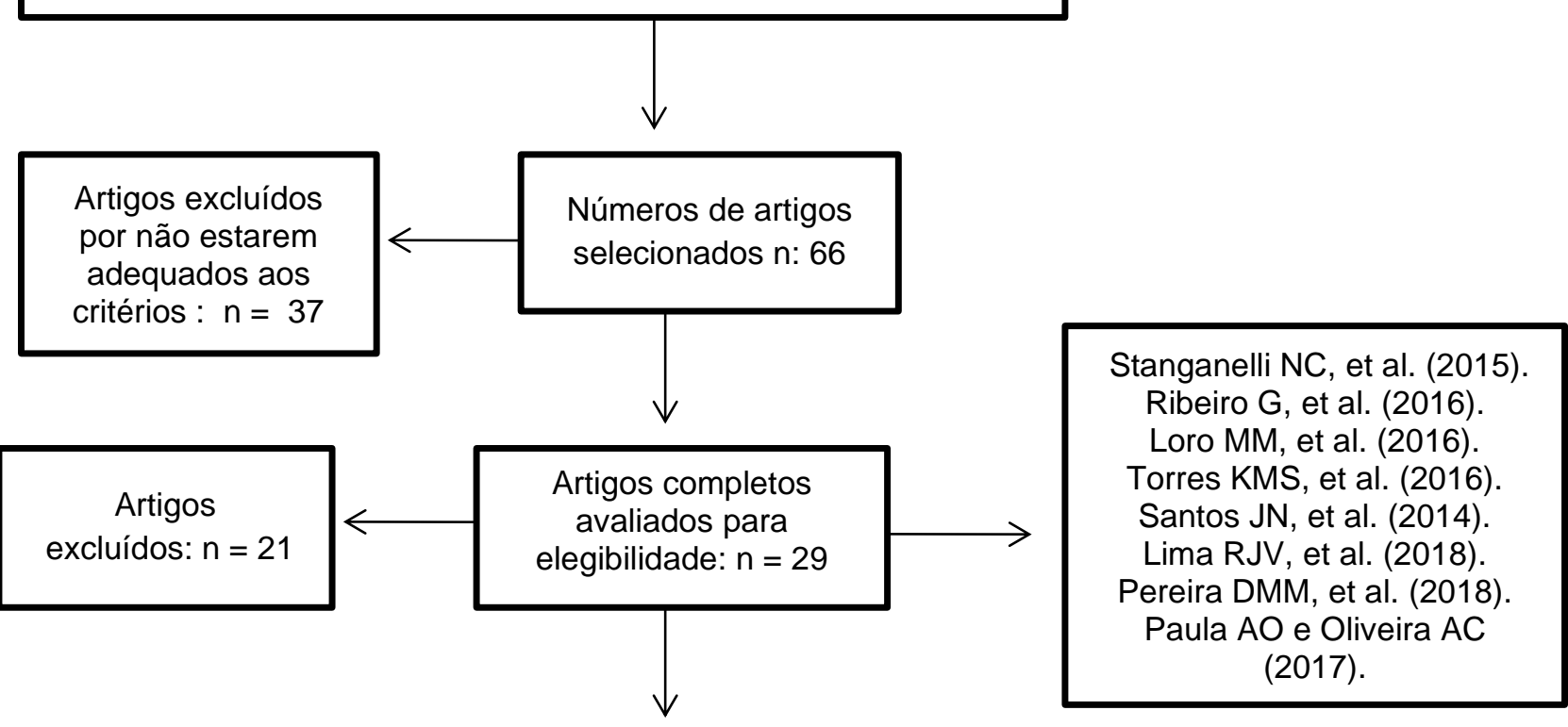

Artigos selecionados: $\mathrm{n}=8$

Fonte: Magalhões MWB, et al., 2019.

Os estudos contiveram suas informações sintetizadas nos tópicos: autor/ano, objetivo, amostra e resultado, os quais foram tabulados e apresentados na Figura 1. Os principais estudos identificados em nossa busca, seus respectivos protocolos e desfechos estão sumarizados no Quadro 1. 
Quadro 1 - Delineamento, métodos e principais desfechos dos estudos selecionados.

\begin{tabular}{|c|c|c|c|}
\hline Autor/ano & Objetivo & Descrição da Amostra & Principais Resultados \\
\hline $\begin{array}{l}\text { RIBEIRO G, et } \\
\text { al. (2016). }\end{array}$ & $\begin{array}{l}\text { Compreender } \\
\text { a prática da biossegurança realizada por } \\
\text { enfermeiros (as) docentes durante a supervisão } \\
\text { de estágio de alunos de cursos técnicos de } \\
\text { enfermagem }\end{array}$ & $\begin{array}{l}11 \text { enfermeiros(as) docentes de duas instituições } \\
\text { da rede federal de educação profissional, } \\
\text { científica e tecnológica na região Sul do Brasil }\end{array}$ & $\begin{array}{l}\text { Ações dos docentes em relação à biossegurança são } \\
\text { influenciadas } \\
\text { pela disponibilidade e acesso aos materiais e } \\
\text { equipamentos. }\end{array}$ \\
\hline $\begin{array}{l}\text { LORO MM, et } \\
\text { al. (2016). }\end{array}$ & $\begin{array}{l}\text { Conhecer a percepção de uma equipe de } \\
\text { enfermagem acerca dos riscos ocupacionais. }\end{array}$ & $\begin{array}{l}24 \text { profissionais da equipe de enfermagem que } \\
\text { atuavam na unidade de urgência e emergência. }\end{array}$ & $\begin{array}{l}\text { Emergiram duas categorias: percepção dos riscos } \\
\text { ocupacionais: um olhar sobre a realidade; e equipamentos } \\
\text { de proteção individual: da percepção à utilização pelo } \\
\text { trabalhador de enfermagem. }\end{array}$ \\
\hline $\begin{array}{l}\text { TORRES KMS, } \\
\text { et al. (2016). }\end{array}$ & 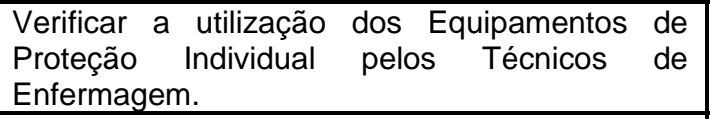 & $\begin{array}{l}20 \text { técnicos de enfermagem, por meio da } \\
\text { aplicação de um questionário semiestruturado } \\
\text { contendo } 9 \text { questões. }\end{array}$ & $\begin{array}{l}20 \text { técnicos de enfermagem e todos alegaram } \\
\text { conhecimento dos equipamentos de proteção individual. }\end{array}$ \\
\hline $\begin{array}{l}\text { SANTOS JN, et } \\
\quad \text { al. (2014). }\end{array}$ & $\begin{array}{l}\text { Verificar a adoção das medidas de } \\
\text { biossegurança em um Ambulatório de } \\
\text { Fonoaudiologia de um hospital escola vinculado } \\
\text { à rede SUS, em Belo Horizonte. }\end{array}$ & $\begin{array}{l}100 \text { atendimentos, escolhidos de modo aleatório, } \\
\text { nas cinco grandes áreas da fonoaudiologia: } \\
\text { Linguagem, Motricidade Orofacial, Audiologia, } \\
\text { Saúde Coletiva e Voz. }\end{array}$ & $\begin{array}{l}4 \% \text { realizaram higienização das mãos antes do } \\
\text { atendimento e } 11 \% \text { utilizaram luvas, } 96 \% \text { utilizaram jaleco } \\
\text { e o mantiveram abotoado ( } 93 \%) \text {, com utilização de } \\
\text { vestimentas adequadas, sapatos fechados ( } 99 \%) \text {, cabelos } \\
\text { presos }(81 \%) \text { e unhas cortadas }(88 \%) \text {. }\end{array}$ \\
\hline $\begin{array}{l}\text { STANGANELLI } \\
\text { NC, et al. } \\
\text { (2015). }\end{array}$ & $\begin{array}{l}\text { Identificar a utilização dos equipamentos de } \\
\text { proteção individual pelos trabalhadores de } \\
\text { enfermagem. }\end{array}$ & $\begin{array}{l}\text { Utilizando-se da observação não-participante e } \\
\text { intencional de } 201 \text { procedimentos por meio de } \\
\text { um checklist. }\end{array}$ & $\begin{array}{l}\text { Verificou-se no Centro Cirúrgico que as luvas de } \\
\text { procedimentos ( } 97 \% \text { ) obtiveram maior adesão, entretanto } \\
\text { sapatos fechados e óculos não foram utilizados nenhuma } \\
\text { vez. }\end{array}$ \\
\hline $\begin{array}{l}\text { LIMA RJV, et al. } \\
\qquad(2018) \text {. }\end{array}$ & $\begin{array}{l}\text { Descrever o conhecimento do conceito de } \\
\text { agentes biológicos e equipamentos de proteção } \\
\text { por profissionais de saúde em ambiente } \\
\text { hospitalar, bem como a utilização dos } \\
\text { equipamentos de proteção individual e coletiva. }\end{array}$ & $\begin{array}{l}\text { Aplicação de questionário a } 67 \text { profissionais de } \\
\text { saúde de um hospital, em Teresina-PI. }\end{array}$ & $\begin{array}{l}84,5 \%, 95,4 \% \text { e } 79,2 \% \text { dos profissionais de saúde } \\
\text { autodeclararam o conhecimento dos conceitos de agentes } \\
\text { biológicos, equipamentos de proteção individual e } \\
\text { coletiva, respectivamente. }\end{array}$ \\
\hline $\begin{array}{l}\text { PEREIRA DMM, } \\
\text { et al. (2018). }\end{array}$ & $\begin{array}{l}\text { Avaliar o nível de conhecimento e adesão das } \\
\text { normas de Biossegurança no setor materno } \\
\text { infantil de um hospital de referência. }\end{array}$ & $\begin{array}{l}54 \text { funcionários dos setores de enfermaria clínica } \\
\text { pediátrica e enfermaria cirúrgica pediátrica. Os } \\
\text { dados foram coletados por meio da aplicação de } \\
\text { questionário com perguntas acerca de assuntos } \\
\text { gerais de Biossegurança. }\end{array}$ & $\begin{array}{l}95 \% \text { dos funcionários declararam saber o que é } \\
\text { Biossegurança, } 59,26 \% \text { dos funcionários declararam que } \\
\text { já receberam algum treinamento em Biossegurança e } \\
51,58 \% \text { desconhecem as Normas Regulamentadoras } \\
\text { relacionadas a prática em Biossegurança. }\end{array}$ \\
\hline $\begin{array}{l}\text { PAULA AO e } \\
\text { OLIVEIRA AC } \\
\quad(2017) .\end{array}$ & $\begin{array}{l}\text { Verificar os aspectos relacionados à percepção } \\
\text { dos profissionais de saúde no que diz respeito à } \\
\text { higienização das mãos (HM), em uma unidade de } \\
\text { pronto-atendimento }\end{array}$ & $\begin{array}{l}\text { Amostra de } 30 \text { profissionais de saúde. A coleta } \\
\text { de dados foi por meio de um questionário, com } \\
\text { questões referentes às características } \\
\text { sociodemográficas, dados relativos ao trabalho e } \\
\text { informações referentes à higienização das mãos } \\
\text { e taxa de adesão a equipamentos. }\end{array}$ & $\begin{array}{l}\text { Os profissionais de saúde atribuem como alto o impacto } \\
\text { das infecções na evolução clínica dos pacientes e a } \\
\text { eficácia da higiene de mãos no controle destas. }\end{array}$ \\
\hline
\end{tabular}

Fonte: Magalhões MWB, et al., 2019. 


\section{DISCUSSÃO}

A biossegurança é a junção de ações direcionadas para a proteção do trabalhador com o propósito de eliminar ou minimizar riscos inerentes às atividades. Deste modo, a biossegurança está inserida no nosso cotidiano como profissional, que não são apenas elementos de uma norma padronizada por um órgão institucional, mas que fazem parte de um planejamento, que visa à saúde dos inseridos nesse processo (FONTANA RT e NUNES DH, 2013).

Os profissionais da área da saúde estão expostos a vírus como hepatite B e C, HIV, herpes entre outros. A contaminação pode ocorrer através de exposições percutânea, mucocutânea, sangue, ou pelo contato com instrumentos ou superfícies contaminadas. Na prática atualmente, nem todos os profissionais de saúde adotam as medidas de biossegurança necessárias à sua proteção durante o manejo de sua atividade, podendo ocasionar agravos a sua saúde e a do paciente (MARTINS CL, et al., 2013).

A Organização Mundial de Saúde (OMS) em 2008, publicou as diretrizes para implementação e avaliação de programas de higienização das mãos em unidades de saúde. Estabeleceram-se cinco estratégias: programas de educação do time de profissionais, disponibilidade de produtos para higienização das mãos, suporte da administração do hospital para o envolvimento do time, feedback dos resultados e lembretes (ROMERO DPM, et al., 2019). As cláusulas de biossegurança, em muitas circunstâncias de assistência, são esquecidas muitas vezes pelos profissionais. O que se espera e se adverte então, é que o profissional esteja apto a empregar as normas de biossegurança sempre que contiver qualquer relação com algum material bem como na cota de cuidados habituais aos pacientes, independente de saber ou não sua condição de saúde (GALLAS SR e FONTANA RT, 2010).

Segundo Ribeiro G, et al. (2016) a prática de biossegurança por profissionais de enfermagem está intimamente ligada ao conhecimento acerca das normas estabelecidas e que no entanto se constitui um valor com potencial de influenciar positivamente os demais profissionais não praticantes. Deste modo, o despreparo para lidar com variados tipos de condições críticas, onde não se analisa a gravidade e a responsabilidade de minimizar um problema, são fatores que podem convir de obstáculos para a adoção de medidas profiláticas na assistência ao paciente.

Loro MM, et al. (2016) afirmam que os profissionais muitas vezes tem a real consciência e percepção dos riscos presente em seu cotidiano, bem como as consequências do não uso das medidas de segurança. Vale ressaltar que a responsabilidade da empresa empregadora no que diz respeito aos aspectos de prevenção para possíveis infecções tem grande interferência na atuação dos profissionais. No entanto, Stanganelli NC, et al. (2015) ressalta que alguns trabalhadores utilizam pretextos para a não utilização dos equipamentos de proteção durante os procedimentos como: sensação de sufoco, incômodo, desconforto, dificuldade na utilização, calor e obrigatoriedade da utilização. Segundo os autores do presente estudo, estes comportamentos influenciam direta e indiretamente na segurança dos trabalhadores e pacientes, colocandoos em situação de risco.

A maioria dos profissionais no que diz respeito às ações de higiene pessoal e utilização dos materiais de biossegurança consideram um esforço alto ou moderado executa-las, e isso indica que essa pratica é um hábito frequente para esses profissionais, o que pode dificultar sua implementação rotineiramente, resultando em baixas taxas de adesão (PAULA AO e OLIVEIRA AC, 2017). O que seria viável a realização de programas de educação permanente continuada para a equipe se abastecer de conhecimento para desenvolver seu papel diante da prevenção de infecções. É importante também destacar que as conferências ou treinamentos sejam feitas por profissional habilitado e em plena noção do assunto e sendo cooperadas e compartilhadas por profissionais de todas as áreas da saúde (REZENDE KCAD, et al., 2012; NAZARIO EG, et al.,2017).

Pereira DMM, et al. (2018) corroboram ao afirmar, que existe uma importância na investigação das ações educacionais que promovam conhecimento não somente dentro do ambiente profissional, mas principalmente nas instituições de ensino para que os alunos possam estar previamente preparados para atuar de forma segura nos ambientes de risco. Outros colaboradores relatam que fatores a exposição aos riscos biológicos em seu ambiente de trabalho e os acidentes relacionados com materiais perfuro cortantes contribuem significativamente para o seu crescimento. Pois estabelecem um problema ocupacional nos serviços de 
saúde, uma vez que favorece danos à integridade mental e física do colaborador, deixando-o vulnerável ao risco de exposição a doenças infecciosas (LIMA SILVA TM, et al., 2019; PAPINI TS, et al., 2014).

Torres KMS, et al. (2016) analisou que todos os profissionais entrevistados em seu estudo obtinham conhecimento do que era e para que serviam os materiais de biossegurança, percebeu-se que só o conhecimento não basta. Santos JN, et al. (2014) verificou a adoção das medidas de biossegurança em um Ambulatório de Fonoaudiologia de um hospital escola vinculado à rede SUS, em Belo Horizonte. 100 atendimentos, escolhidos de modo aleatório, nas cinco grandes áreas da fonoaudiologia: Linguagem, Motricidade Orofacial, Audiologia, Saúde Coletiva e Voz. Observou-se que 4\% realizaram higienização das mãos antes do atendimento e $11 \%$ utilizaram luvas, $96 \%$ utilizaram jaleco e o mantiveram abotoado (93\%), com utilização de vestimentas adequadas, sapatos fechados (99\%), cabelos presos (81\%) e unhas cortadas (88\%). Existe uma potencialização dos fatores riscos de contaminação de profissionais e pacientes. Apesar de os profissionais apresentarem boas práticas quanto à vestimenta e cuidados pessoais, como uso de jaleco, sapatos adequados, cabelos presos e unhas cortadas.

Fatores como jornada exaustiva de trabalho também parece estar relacionado à baixa adesão do cumprimento dos protocolos por parte dos profissionais de saúde. Em um estudo realizado com profissionais de enfermagem ( $n=1215)$ do Hospital das Clínicas da Faculdade de Medicina de Ribeirão, observou-se que a maioria dos profissionais que sofreram acidentes ocupacionais tinham apenas um emprego e faziam uma carga horária semanal de até 36 horas. No entanto, em estudo realizado na mesma instituição, evidenciouse que, para cada hora acrescida na jornada de trabalho, aumentava-se as chances de acidentes percutâneos em 1,03\% (SAMPAIO GB, et al. 2019).

Esses acidentes são relevante devido a possibilidade e facilidade de transmissão de doenças, por meio da interação paciente/profissional de saúde. Sendo os patogênicos, veiculados pelo sangue de maior impacto na saúde do trabalhador, capazes de acarretar outros fatores de desgaste geralmente mais graves que 0 ferimento em si, como a infecção pelos vírus da hepatite B (HBV), da hepatite C (HVC) e da Síndrome da Imunodeficiência Adquirida-AIDS (MOTTA JÁ e BARATA AJSS, 2018).

Em outro estudo, Malaguti-toffano SE, et al. (2012) Observou ainda que 49,3\% das 219 exposições a material biológico notificado ocorreram em profissionais com menos de cinco anos de trabalho e que os profissionais com maior experiência acidentaram menos. No entanto para o autor o tempo de trabalho é incapaz de garantir proteção exacerbada, uma vez que quando o trabalhador adquire mais experiência, logo, o mesmo ganha mais confiança e habilidade prática, devido a isso, julga-se mais protegido e torna-se menos propenso a aderir às medidas de proteção individual.

Para Pimenta FR, et al. (2016) a atuação dos profissionais de saúde, deve ser estabelecida de forma mais complexa a mesma deve requerer a articulação das instituições formadoras com os diferentes níveis de educação, no intuito de estabelecer a construção de processos de educação, não apenas quando os profissionais são contratados, mas permanentemente.

Contudo, Lima RJV, et al. (2018) considera que a adoção de técnicas seguras está intensamente relacionada com a cultura individual, hábitos e conhecimento. Bem como fatores extrínsecos como disponibilidade dessas medidas pela administração hospitalar. Dessa forma, ouso adequado das cláusulas de biossegurança no recinto de trabalho em saúde, agrupado ao conhecimento dos conceitos de equipamentos de segurança, seja individual e/ou coletiva, é qualidade indispensável para a proteção dos funcionários em qualquer que seja a área de desempenho.

Segundo Farias MEL, et al. (2019) os profissionais devem desenvolver um pensamento crítico reflexivo que resulte em uma mudança comportamental dos profissionais no intuito de transformar os mesmos em referências para a equipe, atuando como multiplicadores de informações educativas, visando reforçar 0 desempenho das práticas adequadas em geral. Deste modo, é imprescindível o conhecimento sobre biossegurança em ambiente hospitalar pois os profissionais da área da saúde estão expostos a variados riscos presentes no ambiente de trabalho, enquanto exercem suas atividades, os quais podem gerar acidentes ocupacionais. 


\section{CONSIDERAÇÕES FINAIS}

De acordo com a análise na literatura, foi possível observar que os participantes do estudo tinham consciência e percebiam os riscos em seu cotidiano. Porém, constatou-se também que alguns hospitais não forneciam matérias de biossegurança suficientes para o uso dos profissionais, e que a grande maioria não possuíam os devidos conhecimento sobre a utilização correta dos EPIs e EPCs. Portanto, é essencial que os profissionais sejam treinados e capacitados para utilização correta dos equipamentos de biossegurança, e, a implementação de normas e rotinas a serem seguidas. Proporcionando assim, melhor adesão ao uso dos equipamentos de proteção individual e coletiva, minimizando os riscos de acidentes ocupacionais e infecções para o profissional como para o paciente.

\section{REFERÊNCIAS}

1. ARANTES MC, et al. Acidentes de trabalho com material biológico em trabalhadores de serviços de saúde. Cogitare Enfermagem, 2017; 22(1).

2. ARAÚJO DL, OLIVEIRA JL. Identification and analysis of bacterial presence in diagnostic imaging equipment. Research, Society and Development, 2020; 9(7).

3. CASTRO MR, et al. Condutas da equipe de enfermagem de um hospital universitário em frente à minimização da exposição aos riscos ocupacionais. Revista Brasileira de Pesquisa em Saúde/Brazilian Journal of Health Research, 2013.

4. CUNHA AC, MAURO MYC. Educação Continuada e a Norma Regulamentadora 32: utopia ou realidade na enfermagem?. Revista Brasileira de Saúde Ocupacional, 2010; 35(122): 305-313.

5. FARIAS MEL, et al. Adesão à higiene das mãos antes e após intervenções educativas do dia mundial para higienização das mãos em um hospital universitário. Revista Eletrônica Acervo Saúde, 2019; 11(16): e1354-e1354.

6. FONTANA RT, NUNES DH. Os riscos ocupacionais na concepção dos trabalhadores de uma lavanderia hospitalar. RevEletTrim de enf. 2013.

7. GARCEZ GO, et al. Uso de EPI's pelos profissionais de saúde da atenção básica de um município de Goiás. Revista de Divulgação Científica Sena Aires, 2019; 8(4): 418-426.

8. GALLAS SR, FONTANA, RT. Biossegurança e a enfermagem nos cuidados clínicos: contribuições para saúde do trabalhador. Revista brasileira de enfermagem, 2010; 63(5): 786-792.

9. LORO MM, et al. Desvelando situações de risco no contexto de trabalho da Enfermagem em serviços de urgência e emergência. Escola Anna Nery, 2016, 20(4).

10. LIMA RJV, et al. Agentes biológicos e equipamentos de proteção individual e coletiva: conhecimento e utilização entre profissionais. Revista Prevenção de Infecção e Saúde, 2018; 3(3).

11. LIMA SILVA TM, et al. Vestimentas dos profissionais da saúde: riscos e cuidados necessários. Revista de Administração em Saúde, 2019; 19(74).

12. MOTA EC, et al. Higienização das mãos: uma avaliação da adesão e da prática dos profissionais de saúde no controle das infecções hospitalares. Rev Epidemiol Control Infect, 2014; 4(1): $12-17$.

13. MALAGUTI-TOFFANO SE, et al. Adesão às precauções-padrão entre profissionais da enfermagem expostos a material biológico. Revista Eletrônica de Enfermagem, 2015; 17(1): 131-5.

14. MARTINS CL, et al. Equipamentos de proteção individual: a perspectiva de trabalhadores que sofreram queimaduras no trabalho. Rev Enferm UFSM, 2013; 18.

15. MOTTA JA, BARATA AJSS. Gestão hospitalar: aspectos de saúde ocupacional associados a uma instituição. Revista Saúde e Meio Ambiente, 2018; 7(3): 42-57.

16. NAZARIO EG, et al. Riscos ocupacionais e adesão a precauções-padrão no trabalho de enfermagem em terapia intensiva: percepções de trabalhadores. Revista Brasileira de Saúde Ocupacional, 2017; 42.

17. PEREIRA DMM, et al. Conhecimento e adesão às práticas de biossegurança em um hospital de referência materno infantil. Pará Research Medical Journal, 2018; 1(3): 0-0.

18. PIMENTA FR, et al. Care and specialized clinical follow-up of nursing professionals who have been victims of accidents with biological material. Rev Esc Enferm USP, 2016; 12, 47(1): 198-204.

19. PAPINI TS, et al. Avaliação da vestimenta utilizada como equipamento de proteção individual pelos aplicadores de malationa no controle da dengue em São Paulo. Cad. Saúde Pública, Rio de Janeiro, 2014; 16.

20. PAULA AO, OLIVEIRA AC. A percepção dos profissionais de saúde em relação à higienização das mãos. Revista de Pesquisa: Cuidado é fundamental online, 2017; 9(2): 321-326.

21. RIBEIRO G, et al. Práticas de biossegurança no ensino técnico de enfermagem. Trabalho, Educação e Saúde, 2016; 14(3): 871-888.

22. ROMERO DMP, et al. Efeitos da implementação de um programa de educação de higienização das mãos entre profissionais de uma UTI: análise de séries temporais interrompidas. J. bras. Pneumol. São Paulo, 2019; 45(5): e20180152.

23. REZENDE KCAD, et al. Adesão à higienização das mãos e ao uso de equipamentos de proteção pessoal por profissionais de enfermagem na atenção básica em saúde. Ciência, cuidado e saúde, 2012; 11(2): 343-351. 
24. SANTOS JN, et al. Condutas de biossegurança em ambulatório de fonoaudiologia da rede SUS. Distúrbios da Comunicação, 2014; 26(1).

25. STANGANELLI NC, et al. A utilização de equipamentos de proteção individual entre trabalhadores de enfermagem de um hospital público. Cogitare Enfermagem, 2015; 20(2).

26. SOUZA ELV, et al. Uso dos equipamentos de proteção individual em unidade de terapia intensiva. Revista de Enfermagem Referência, 2011; 3(4): 125-133.

27. SAMPAIO GB., et al. Educação Permanente e o Processo Formativo dos Profissionais da Saúde: Entrelace de Saberes. Revista Eletrônica Acervo Saúde, 2019; 25: e630-e630.

28. SILVA PIRES YM, et al. Saúde do trabalhador em ambiente hospitalar: mapeando riscos e principais medidas de biossegurança. Revista uningá, 2019; 56(2): 115-123.

29. TORRES KMS, et al. Use of individual protection equipment by nursing technicians/Uso de equipamentos de proteção individual por técnicos de enfermagem/Personal uso para el equipo de protección de enfermeria. Revista de Enfermagem da UFPI, 2016; 5(4). 